\title{
Society for Research on Nicotine and Tobacco
}

\author{
KENNETH A. PERKINS ${ }^{1}$, NEAL BENOWITZ ${ }^{2}$, JACK \\ HENNINGFIELD ${ }^{3}$, PAUL NEWHOUSE ${ }^{4}$, OVIDE POMERLEAU ${ }^{5}$ \\ \& GARY SWAN ${ }^{6}$
}

${ }^{1}$ Associate Professor of Psychiatry, Western Psychiatric Institute $\mathbb{E}$ Clinic, University of Pittsburgh School of Medicine, 3811 O'Hara Street, Pittsburgh, PA 15213; '2Professor of Medicine, University of Califormia at San Francisco, Building 30, 5th Floor, 1001 Potrero Avenue, San Francisco, CA 94110; ${ }^{3}$ Chief, Clinical Pharmacology Branch, Addiction Research Center, National Institute on Drug Abuse, P.O Box 5180, Baltimore, MD 21224; ${ }^{4}$ Associate Professor of Psychiatry, Clinical Neuroscience Research Unit, University of Vermont, 1 South Prospect Street, Burlington, VT 05401-1195; ${ }^{5}$ Professor of Psychiatry, University of Michigan, Williamsburg Square, 475 Market Place, Suite L, Ann Arbor, MI 48108 and ${ }^{6}$ Health Sciences and Policy Program, SRI International, 333 Ravenswood Ave, Menlo Park, CA 94025, USA

\begin{abstract}
The proceedings of the inaugural scientific meeting of the Society for Research on Nicotine and Tobacco (SRNT) are summarized. The primary objective of the meeting was to foster the exchange of information on the effects of nicotine and tobacco use, as well as factors which influence their use, drawing from biological, behavioral and social sciences. Much of this research can be viewed as a tale of "two" drugs-nicotine as a key to an important public health problem, and nicotine as a classical tool of physiological and pharmacological research. A historical overview of research on "both" drugs is provided first. Public policy alternatives for reducing the prevalence of tobacco use have been derived in part from basic and clinical research results and are briefly outlined. Evidence for genetic determinants on nicotine use and effects is presented using data from twin studies and from molecular genetic research with humans and animals. Consistent with this research, there is evidence of individual differences in pharmacokinetics and effects of nicotine, which could account for differences in smoking behavior and nicotine dependence. Finally, recent developments in the therapeutic uses of nicotine and novel nicotinic agonists with schizophrenia, Alzheimer's disease, Parkinson's disease, Tourette's syndrome and ulcerative colitis are presented. Overall, the research presented at the meeting demonstrated the vast diversity of areas of study involving nicotine and tobacco, as well as the rich opportunities for cross-communication among researchers from different disciplines.
\end{abstract}

Correspondence to: Kenneth A. Perkins, WPIC, University of Pittsburgh School of Medicine, 3811 O'Hara Street, Pittsburgh PA 15213, USA. Tel.: (412) 624-1716; Fax: (412) 624-6018. 


\section{Introduction}

Research on nicotine and tobacco has grown in volume as well as widened in scope, especially over the past decade. With this growth has come a splintering of the field, such that this research is now presented at a myriad of meetings, ranging from those focusing on molecular biology to others emphasizing public health. Moreover, nicotine and tobacco too often comprise a very small component of these meetings. Given this divergence, it is increasingly uncommon for nicotine and tobacco researchers from these different disciplines to come together and share their findings.

The Society for Research on Nicotine and Tobacco (SRNT) was organized in 1994 to provide a forum for the exchange of information across the entire spectrum of research concerning nicotine and tobacco use and effects. The Society held its inaugural scientific meeting 2425 March, 1994 in San Diego CA. More than 220 registrants from nine countries attended the forums, symposia, Master Lecture and paper and poster sessions. Selected individual paper and poster abstracts are presented elsewhere in this issue. A summary of the other proceedings follows.

Past, present, and future perspectives on nicotine research

A new society can best gain its bearings by placing itself in historical context. Such a context was provided for the audience by SRNT President Ovide Pomerleau, Edward Domino, John Hughes and former US Surgeon General Jesse Steinfeld. In contrast to the current widespread focus on the health impact of nicotine and tobacco use, nicotine was initially used as a tool in pharmacology and physiology research earlier in the century. For example, in 1905 John Langley reported that low doses of nicotine stimulated and high doses of nicotine "paralyzed" autonomic ganglia. Moreover, as early as 1901 Langley's research on nicotine had already led him to use the term "receptive substance" to characterize nicotine's effects at the neuromuscular junction. Hence, the birth of the term "receptor". Similarly, Sir Henry Dale reported in 1914 that nicotine could be used to differentiate cholinergic receptors into muscarinic and nicotinic types. Because of such breakthroughs, nicotine was known for a time as the "pharmacologic scalpel". Although many of the basic questions remain, such as what effects nicotine has in the brain, most of the tools have changed, including use of positron emission tomography (PET) scanning of neural activity during nicotine exposure (Nagata $e t$ al., 1995). Further technological developments will only improve the precision with which we understand nicotine's actions.

Knowledge of nicotine and tobacco use and effects, of course, predates the 20 th century. Nicotine played important roles in indigenous native American culture for more than 5000 years, including its use in religious and medicinal rituals, as recorded in native art. Among the most enthusiastic European proponents of tobacco use was Jean Nicot, French ambassador to Portugal, in whose honor Catherine de Medici, the 16th century French Queen, named the tobacco plant (Tabacum nicotiana). Misgivings about the health effects of tobacco use were sounded as early as the 17 th and 18 th centuries but were overshadowed by the lucrative commercial value and tax receipts resulting from the tobacco trade (a portent of things to come). The advent of the cigarette-rolling machine, the safety match, improved tobacco blends and advertising all led to the tremendous growth of tobacco smoking, and hence tobacco production, around the turn of the 20th century. Despite western societies' acknowledgement of health risks associated with tobacco use over the past 30 years, world-wide tobacco production has continued to increase, with the result of increasing prevalence of tobacco use in developing countries.

An overview of the current state of smoking cessation treatment research was then presented. Although the number of treatment studies focusing on cessation of tobacco use has increased almost exponentially since 1970 (Shiffman, 1993), there have been only a few legitimate breakthroughs in the development of efficacious interventions. Nicotine replacement therapy is certainly one but has proved to be less than a panacea (Hughes, 1993). Behavioral treatments have also demonstrated efficacy, but they too fail more often than they succeed with smokers trying to quit. Future research into interventions for smoking cessation will not bear fruit without undertaking a fresh examination of nicotine and tobacco reinforcement and the basic biological and behavioral underpinnings of tobacco depen- 
dence. These treatments will also have to be tailored to smokers who have other substance use or psychiatric problems, such as alcohol dependence or affective disorder, as well as to under-served smokers for whom traditional treatments are unavailable or inadequate.

A different approach society can take to reduce the prevalence of smoking is to support governmental involvement in regulating tobacco. Despite the logic of this approach, there are clear practical obstacles limiting the likelihood of success. Steinfeld recounted his own experiences in fostering the rights of US non-smokers in 1971, leading to his dismissal from US government service soon after. He further outlined examples of how political expediency can adversely influence the actions of even the most sympathetic law-makers when it comes to attempts to curb the health impact of smoking. Overall, this session served to underscore the breadth of past and current research into nicotine and tobacco and to highlight how political, economic and social issues surrounding tobacco often re-appear across the decades and even centuries.

\section{Regulation of nicotine products in the US: research issues}

Taking a more optimistic stance in terms of potential government involvement in control of nicotine products, presenters in the subsequent forum emphasized the relevance of findings from basic and clinical research to the development of strategies for limiting access to tobacco or to nicotine exposure. Inquiries regarding the appropriate means to regulate nicotine and tobacco products by the US Food and Drug Administration, the US Federal Trade Commission and the US Congress in 1994 raised a broad range of potential policy issues (Henningfield \& Benowitz, 1995; Kozlowski \& Henningfield, 1995). Importantly, many of these issues can be evaluated in the context of empirical research. Jack Henningfield, Lynn Kozlowski, Neal Benowitz, John Slade, John Hughes and Charles Gruder described a variety of approaches US and state governments can take to regulate these products, as well as the research supporting these approaches.

The driving force behind reappraisal of current nicotine and tobacco regulatory approaches is that the United States (along with the rest of the world) is making unacceptable progress, or even losing ground, in combating the impact of tobacco use on public health, especially among American youth and in developing countries. In the United States, it appears that tobacco-related deaths will remain at the level of 400000 per year or more for some time to come. Particularly discouraging is that tobacco use among youth appears to have increased somewhat over the past decade. Worldwide, Peto and colleagues (1992) estimate that half of all smokers will die prematurely due to their smoking and that tobacco-related deaths will increase from 3 million per year in the 1990 s to 20 million per year by the 2020s unless there are substantial decreases in smoking behavior. Present trends suggest that tobacco use has flattened after a period of decline in the United States and is still on the rise world-wide. It can thus be argued that present regulatory approaches provide little in the way of effective barriers to tobacco use. At the same time, these approaches currently provide substantial barriers to the widespread use of effective nicotine-replacement medication designed to assist in the cessation of tobacco use.

Among the actions recommended was possible revisions in the US Federal Trade Commission (FTC) labeling of cigarettes with respect to their delivery of nicotine, as well as tar and other constituents (Henningfield, Kozlowski \& Benowitz, 1994). Human laboratory and field research has demonstrated for some time that stated nicotine yields of cigarette brands do not reflect actual nicotine exposure by the smoker due to the disparity between FTC machine smoking vs. actual human smoking of cigarettes. An additional, longer-term strategy is to gradually reduce the nicotine levels in cigarettes to the point that they would not be considered pharmacologically addicting (Benowitz \& Henningfield, 1994). Studies with humans and animals have begun to identify the doses at which nicotine becomes discriminable and reinforcing, providing a theoretical cut-off for cigarette yields. A third, more extreme approach would be to severely restrict access to nicotine in all forms, including tobacco, by making it available by prescription only. This position is supported by the view that tobacco companies function in many respects as pharmaceutical companies, especially in terms of their product development and manufacturing procedures (e.g. Slade, 1995). Thus, their products should undergo the same regulatory evaluation 
as other pharmaceutical products. Complementing this approach is a strategy to make nicotine replacement medications more readily available, such as by allowing over-the-counter access. Finally, the importance of state, as opposed to US federal, efforts to regulate and tax tobacco products was outlined, using California as an example. Despite political obstacles to maintaining the program (echoing comments by previous speakers), proponents have shown that such a program can lead to reduced prevalence of smoking, especially among youth, and that tax revenues can be used to enhance the support of tobacco-related research.

\section{Genetic influences on use and effects of nic- otine and tobacco}

Tobacco products are available virtually throughout the world, and nearly all teens experiment with tobacco use. Yet, in most societies, fewer than half of adults are regular users of tobacco. Thus, the issue of why some people are at heightened risk for the adoption and/or maintenance of smoking remains of enormous concern to public health officials as well as to those responsible for allocation of increasingly scarce health care resources. Evidence from several lines of research suggests that the use and effects of nicotine or tobacco smoking is influenced in part by genetic factors. Gary Swan, Andrew Heath, Allan Collins, Ernest Noble, and Gerald McClearn presented recent studies in support of this notion.

More than 20 studies have examined tobacco use in monozygotic and dizygotic twins, demonstrating that both smoking initiation and persistence are regulated by genetic factors. One of the more recent studies used information on smoking history for 4775 pairs of US twins surveyed in 1967-69 and then again in 1983-85 (Carmelli et al., 1992). The large sample size permitted classification by smoking status, smoking intensity (number of cigarettes per day), and smoking cessation at follow-up. Genetic influence (i.e. greater concordance in monozygotic vs. dizygotic twins) was most strongly seen at the tails of the distribution of smoking intensity (low and high number of cigarettes per day) and was also observed for cessation at follow-up. Other twin studies from Finland, Sweden, Australia, England, and the United States provide heritability estimates (i.e. proportion of total variance in risk attributable to genetic effects) in the range 28$83 \%$ for smoking initiation and $53-71 \%$ for persistence, with the genetic influences on each being independent of the other (Heath et al., 1995). Notably, findings are as great in women as in men and appear to be comparable to those from studies on alcoholism. At least some of the genetic contributions to risk of becoming a smoker may be mediated through differences in personality (e.g. novelty seeking, social conformity) and educational attainment. On the other hand, these variables are only weakly predictive of smoking persistence, leaving unanswered the question of how genetic influences on persistence arise. In addition, the extent to which genetic differences in initial sensitivity or in rate of acquisition of tolerance to nicotine may influence risk of nicotine dependence, as well as probability of becoming a long-term persistent smoker, remains a critical issue. Further twin research should: incorporate refined indices of nicotine dependence (such as laboratory measures of nicotine sensitivity and metabolism), examine potential moderating influences of gender, and investigate genetic influences on the joint use of tobacco, alcohol and other substances.

Animal research has addressed many of these issues with impressive precision. Studies by Collins and others have attempted to identify components of the dependence process that are regulated by genetic factors (e.g. Marks et al., 1991). Using inbred mouse strains, selectively bred mouse lines and recombinant inbred strains, they have shown that genetic factors regulate sensitivity to a first dose of nicotine, the development of acute and chronic tolerance to nicotine, and preference for nicotine-containing solutions in a choice test. Initial sensitivity, related to number of brain nicotinic receptors, also seems to be a predictor of tolerance and self-administration. Quantitative trait loci analyses have identified other candidate genes that are currently being investigated.

Complementing this animal work are very recent molecular genetic studies in humans suggesting the importance of certain gene variants of the dopaminergic system in smokers (Noble, et $a l .$, 1994). For example, Noble has very recently found a higher prevalence of gene variants of the D2 dopamine receptor (DRD2) among active and ex-smokers compared with never-smokers in 
a sample of 169 Caucasian women (100 neversmokers, 69 current or ex-smokers) and 164 Caucasian men ( 96 never-smokers, 68 current or ex-smokers). Analysis showed that male smokers carrying the Taq I DRD2 A1 allele (A1/A1 or A1/A2 genotype), as opposed to those carrying the A2 allele (A2/A2 genotype only), began to smoke regularly at a significantly earlier age (15.9 vs. 18.3 years). However, there was no difference due to genotype for female smokers. To attempt to better understand this gender difference, the influence of body mass index (BMI) was considered. Of the females, 92 were non-obese and 77 were obese, while of the males 85 were non-obese and 79 were obese. No significant differences in BMI were found among the $A 1$ and $A 2$ allele non-smoker females and males. However, significant differences were found among the four groups of smokers. Specifically, BMI was lower in A1 allele compared to A2 allele female smokers, while this difference was reversed for male smokers. Similar results were observed in analyses of Intron 6/Exon 7 alleles. Thus, genetic analyses of smoking should consider the moderating influences of other heritable conditions, such as obesity.

In evaluating the current state of this genetic research McClearn noted that, until recently, there were only two general approaches to studying pharmacogenetics: the single gene and quantitative genetic approaches. The single gene approach is attractive because success in identifying a single locus frequently points to a possible mechanism. However, in many important phenotypes there appears to be no single gene that accounts for variability in the population. An alternative "differential" model (McClearn, 1993), based on quantitative genetic theory, accounts for multiple genetic and environmental influences impinging on a common "causal field", leading to the phenotype under examination. Principal features displayed by the model are the widespread ramifications of allelic differences at a single locus (pleiotropy), the influence of multiple loci on complex phenotypes (polygeny), and the genetic and environmental origins of correlations among phenotypes. Since every human is unique in terms of genetics and environmental experience, individual variability is a fundamental feature of any biological system, not just a reflection of "error". Findings from genetic studies can perhaps be more clearly understood in the context of this "differential" model. Further advances in behavioral and molecular genetic research will likely provide a clearer understanding of the biobehavioral bases of nicotine dependence and, consequently, aid in the development of improved methods for assessment and treatment of dependence.

\section{Individual differences in nicotine dispo- sition and effects in humans}

As suggested in the genetic research previously discussed, individual differences in the initiation and persistence of tobacco use, as well as in the development of adverse health effects of smoking, are well recognized. The bases for these individual differences are as yet unknown but could involve differences in metabolism and/or pharmacodynamics of nicotine. Neal Benowitz presented a Master Lecture describing recent research findings on individual differences in nicotine disposition and effects.

The pattern of nicotine metabolism has been nearly completely characterized, with $90 \%$ of a dose of nicotine recoverable in the urine. However, there is considerable individual variability in patterns of metabolism, which could contribute to individual variability in susceptibility to nicotine addiction in two ways. First, the rate of nicotine metabolism could influence how much people smoke. For example, a person who metabolizes nicotine quickly may need to smoke more to achieve a particular level of nicotine than does a person who metabolizes nicotine more slowly. Secondly, the pattern of nicotine metabolites (including cotinine, nicotine iminium ion, beta-nicotyrine, nornicotine) that is generated could affect smoking behavior since some of these metabolites may be pharmacologically active.

Among smokers, there are four-fold differences in nicotine clearance. Using deuteriumlabeled nicotine and cotinine, the disposition kinetics of nicotine have been studied in a variety of patient populations. A comparison of smokers and non-smokers indicated that nicotine metabolism is slightly faster in non-smokers (Benowitz \& Jacob, 1993). Thus, metabolic tolerance does not appear to be a factor in the natural history of tobacco addiction.

Conversion of nicotine to its major metabolite, cotinine, has been the subject of recent research into individual differences. In a group of 20 
smokers it was determined that, on average, $72 \%$ (range 55-92\%) of nicotine is converted to cotinine (Benowitz \& Jacob, 1994). Using data from several sources a factor was derived that relates cotinine concentration to daily nicotine intake. This factor averages 0.08 , such that a typical plasma cotinine level of $300 \mathrm{ng} / \mathrm{ml}$ corresponds to a daily intake of $24 \mathrm{mg}$ nicotine. Yet, one individual has been found to convert only $9 \%$ of nicotine to cotinine (Benowitz, Sachs \& Jacob, 1995). This individual also had abnormally slow elimination of nicotine (three-fold prolongation of nicotine half-life). The biological consequences of this deficient $\mathrm{C}$-oxidation of nicotine is unclear but could be considerable.

Ethnic differences in nicotine metabolism have been hypothesized to contribute to differences in health effects and/or susceptibility to nicotine addiction in blacks vs. whites. Notably, cotinine levels per cigarette smoked are significantly higher in blacks, and blacks have higher rates of lung cancer at any given level of smoking, compared with whites. To examine whether metabolism of nicotine or cotinine could be involved, labeled nicotine and cotinine infusions were administered to 40 black and 39 white smokers matched for age, gender and self-reported cigarette consumption (Benowitz et al., 1995). Nicotine clearance and percentage conversion to cotinine were similar between blacks and whites, but cotinine clearance was significantly slower (and cotinine half-life longer) in blacks. Intake of nicotine per cigarette was also higher in blacks. These data clarify higher cotinine levels per cigarette smoked in blacks, although their relationship to differential rates of nicotine addiction and health risks are still unclear. Collaboration between clinical pharmacologists and geneticists may clarify many of the mechanisms accounting for these individual differences in nicotine metabolism.

Aside from individual differences in nicotine metabolism, variability in magnitude of effects of nicotine may also impact on nicotine addiction and health effects. Increasingly clear is the fact that effects of nicotine depend on the rate of dosing (Porchet et al., 1987) Thus, rapid dosing results in greater effects because of the resulting higher arterial concentrations of nicotine, compared with slow dosing. Pharmacodynamic studies have also focused on the relationship between plasma nicotine concentrations and effects over time, with particular emphasis on the develop- ment of tolerance. Tolerance, which can be defined as reduced magnitude of drug effect with repeated exposure, is considered a key to the onset of physical dependence. Pharmacokineticpharmacodynamic modeling has been used to characterize acute tolerance to specific nicotine effects (Porchet, Benowitz \& Sheiner, 1988). For example, this method has determined that acute tolerance to the heart rate acceleration effects of nicotine has a half-life of 35 minutes. Furthermore, this model indicates that at a steady state plasma nicotine concentration of $25 \mathrm{ng} / \mathrm{ml}$, typical of levels observed in smokers, the heart rate acceleration effect of nicotine is only $20 \%$ of that observed in the absence of tolerance. These results mirror heart rate effects of smoking over the course of a day, with substantial increases due to the first few cigarettes of the day, followed by plateauing in the face of rising nicotine levels. Similar pharmacodynamic parameters have been observed for blood pressure effects and the epinephrine releasing effects of nicotine. In contrast, tolerance to nicotine's effects on energy expenditure develops more rapidly, suggesting a different mechanism of action for tolerance to this effect vs. cardiovascular effects. Use of these methods to examine individual differences in tolerance development may provide a mechanism or marker of differential susceptibility to nicotine addiction.

\section{Therapeutic potential of nicotine and nico-} tinic agonists

As previously noted, the SRNT meeting opened with a recounting of some of the history of nicotine research, and it was pointed out that nicotine has long been a useful tool for researchers interested in probing the nervous system. Perhaps nowhere is this vein of research more alive and well than in the study of possible therapeutic uses of nicotine. Although the health risks associated with its intake via tobacco products has tended to tarnish society's view of nicotine, it is important to recognize that nicotine may have therapeutic potential with a number of disease states. Paul Newhouse, Robert Freedman, Paul Sanberg, Marc Silverstein and Steven Arneric described developments in the use of nicotine, nicotinic antagonists and novel nicotinic agonists in the investigation and treatment of neuropsychiatric and gastrointestinal conditions. 
Tobacco smoking is extremely prevalent among those with the psychiatric disorder of schizophrenia. Part of the reason they smoke so heavily may be to improve ability to screen out irrelevant sensory information. Schizophrenics show impairment in this ability, which may be related to an impairment of inhibitory mechanisms that act to decrease attention to repeated stimuli (i.e. sensory gating). One of the neuronal mechanisms responsible for such gating involves the activation of nicotinic receptors in the hippocampus. These receptors have been found to be diminished in schizophrenics at autopsy. Investigations in living patients have shown that schizophrenics and $50 \%$ of their first-degree relatives show deficient sensory gating to repeated auditory stimuli (P50). Nicotine transiently restores normal suppression (gating) of P50 responses, with effects lasting 15-30 min. Pretreatment of subjects with the ganglionic-type (C6) nicotinic antagonist mecamylamine (10 $\mathrm{mg}$ ) did not block the restoration of gating, suggesting that this effect of nicotine may be mediated through bungarotoxin-type nicotinic receptors. Studies of the hippocampal nicotinic receptor that may be responsible for the gating effects show that the alpha-bungarotoxin sensitive receptor consists solely of alpha-7 subunits. These receptors occur on interneurons in the dentate hilus and CA3 regions of the hippocampus. Recently, the gene encoding the alpha-7 nicotine receptor unit has been localized to 15q14. Preliminary studies of families with schizophrenia and the P50 gating abnormality showed linkage to this chromosomal location. Therefore, medications to improve sensory gating in these patients may be an attractive therapeutic target (Freedman et al., 1994).

Nicotine may also be efficacious in augmenting neuroleptic therapy in patients with Tourette's syndrome (TS). TS usually begins in childhood and is characterized by multiple motor and vocal tics. Treatment of choice is dopamine antagonists such as haloperidol. However, some patients do not fully respond to such agents and full suppression is not possible in others due to dose-limiting side-effects. In animals, nicotine markedly potentiates the cataleptic effects of neuroleptics while not producing these effects when administered alone (Emerich et al., 1991). This action appears to be mediated through interactions at striatal D2 receptors as nicotine does not potentiate cataleptic effects of selective
D1 antagonists. Drawing from this research, Sanberg provided nicotine polacrilex (gum) to TS patients (adults and children) who had only partially responded to haloperidol, finding significant relief of symptoms as soon as $20 \mathrm{~min}$ after nicotine administration. Improvement was observed in both frequency and severity of motor and vocal tics as well as in improved concentration and attention. Difficulties in administration and compliance with the gum have led to similar research with transdermal nicotine, which has resulted in improvements similar to that from gum, especially in non-smokers. Unexpectedly, some patients showed prolonged suppression of symptoms after a single exposure to nicotine, with amelioration lasting up to several weeks (Silver \& Sanberg, 1993). While the mechanism(s) behind this augmentative effect of nicotine remains unclear, the caudate nucleus appears critical, as direct injections into the caudate can replicate the effect of systemic administration in rats and lesioning the caudate obliterates the response.

Interest in the role of nicotinic systems in the cognitive disorder of Alzheimer's disease (AD) increased after the development of technology to map central nervous system (CNS) nicotinic receptors. Subsequently, AD patients have been shown to have a marked reduction in brain nicotinic receptor density compared to age-matched controls. Newhouse examined the potential effects of nicotine receptor loss on cognitive function by administering the nicotine antagonist mecamylamine to young and old normals, AD and Parkinson's disease (PD) patients. Mecamylamine produced dose-related impairment in acquisition of new verbal or non-verbal information, with a significant shift to the left in dose sensitivity with age and disease state (Newhouse et al., 1994). PD patients did not show the sensitivity to nicotinic blockade that $\mathrm{AD}$ patients did, despite a prior finding that PD patients also show loss of nicotinic receptors. Dopamine-agonist treatment of such patients may have prevented effects of mecamylamine. Studies with intravenous nicotine in $\mathrm{AD}$ patients have shown that nicotine can improve cognitive function in many of the same cognitive domains, with a dose-related decline in verbal learning errors and increase in long-term recall (Newhouse et al., 1993). These results suggest that nicotinic modulation may alleviate cognitive impairments in various dementing disorders which show loss 
of nicotinic receptors. Nicotine is unlikely to be an ideal candidate for this task due to its low therapeutic index but other, more selective, agonists may be more useful, as discussed below.

Illustrative of the diversity of sites of nicotine's actions in the body is preliminary evidence of its role in inflammatory bowel disease. Silverstein reported that in the 1980 s there were case reports of patients with ulcerative colitis (UC) in which symptoms began immediately after smoking cessation or improved after resuming smoking or chewing nicotine gum. An early case-control study demonstrated that current smokers had reduced risk (odds ratio (OR) of 0.31 ) and former smokers had slightly increased risk (OR $=1.16$ ) of being diagnosed with $U C$, compared with the general population. Silverstein and colleagues (1994) have also performed a case-control study, controlling for factors known to be associated with $\mathrm{UC}$, such as race, religion and socio-economic status. Results showed that current smokers had an adjusted OR of 0.22 and former smokers had an adjusted OR of 1.6 for UC. Risk of onset for UC is substantially increased shortly after quitting smoking (OR of 7.5 in first 2 years after quitting but 0.446 years after quitting). Initial clinical trials with nicotine gum in UC have shown improvement in about half of patients when it is added to their normal therapeutic regimen. A recent double-blind study of 6-week treatment with nicotine patches has also shown significant improvements in global clinical and histological appearance, less symptoms and more complete remissions. In contrast with this relationship between smoking and UC, patients with another major inflammatory bowel disease, Crohn's disease (CD), are more likely to be smoking at the onset of symptoms $(O R=3.7)$ and to show therapeutic improvement after smoking cessation (Silverstein et al., 1989). Thus, further study of nicotine's gastrointestinal mechanisms of action is clearly needed.

The research reviewed clearly indicates therapeutic potential for nicotine. However, development of novel nicotinic agonists may provide for greater efficacy and reduced side-effects, as presented by Arneric. With increased understanding of the molecular biology of the nicotinic acetylcholine receptor (NAR), new molecules are being developed which have enhanced selectivity for nicotinic receptor subtypes or which may be allosteric modulators of nicotinic receptor func- tion. One of these is ABT-418, a potent and selective NAR agonist which appears to be relatively selective for alpha-4 beta-2 NAR subrype (Arneric et al., 1994). It has little activity at the bungarotoxin-labeled NAR site (alpha-7) or at the neuromuscular junction. Mecamylamine blocks effects of ABT-418 on activating NAR channel currents in PC12 cells, suggesting that ABT-418 is a ganglionic-type nicotinic agonist. Animal studies show that ABT-418 has positive effects on inhibitory avoidance at lower doses than nicotine (which is blocked by mecamylamine), restores normal performance in septallesioned rats, enhances primate performance on delayed matching-to-sample tasks, and produces anxiolysis similar to non-benzodiazepine anxiolytics (Garvey et al., 1994). However, unlike nicotine, ABT-418 does not produce changes in free-running EEG. Other nicotinic agonists under development include GTS-21, an anabaseine derivative which may have activity at alpha-7 NARs and may be neuroprotective. Another agonist, S-1663, appears to be selective for alpha-3 beta-4/2 NARs and may selectively enhance dopamine release and reduce neuroleptic-induced catalepsy. Aside from their potential therapeutic efficacy with Alzheimer's and Parkinson's diseases, novel NAR may have potential in treating benign familial neonatal convulsions and as analgesics.

Understanding the regulatory role of nicotine receptors and their underlying molecular and cellular biology is increasing rapidly. This research may lead to a new era in the development of selective nicotinic agents for a variety of disease states.

\section{Conclusion}

The inaugural scientific meeting of the Society for Research on Nicotine and Tobacco was organized to foster the exchange of information on the effects of nicotine and tobacco use, as well as factors which influence their use, drawing from biological, behavioral and social sciences. Much of this research can be viewed as a tale of "two" drugs--nicotine as a key to an important public health problem, and nicotine as a classic tool of physiological and pharmacological research. Overall, the research and policy issues presented at the meeting demonstrated the vast diversity of areas of study involving nicotine and tobacco, as well as the rich opportunities for cross-com- 
munication among researchers from different disciplines.

\section{References}

Arneric, S. P., Sullivan, J. P., Briggs, C. A. et al. (1994) ABT-418: a novel cholinergic ligand with cognition enhancing and anxiolytic activity. I. In vivo activity, Journal of Pharmacology and Experimental Therapeutics, 270, 310-318.

BenowitZ, N. L. \& HeNnINGFIELD, J. E. (1994) Establishing a nicotine threshold for addiction, New England Fournal of Medicine, 331, 123-125.

Benowitz, N. L. \& JACOB, P. (1993) Nicotine and cotinine elimination pharmacokinetics in smokers and nonsmokers, Clinical Pharmacology and Therapeutics, 53, 316-323.

BENOwITZ, N. L. \& JACOB, P. (1994) Metabolism of nicotine to cotinine studied by a dual stable isotope method, Clinical Pharmacology and Therapeutics, 56, 483-493.

Benowrtz, N. L., SACHS, D. \& JaCob, P. (1995) Deficient C-oxidation of nicotine, Clinical Pharmacology and Therapeutics, 57, 590-594.

Benowitz, N. L., Perez-Stable, E., Herrera, B. \& JACOB, P. (1995) African American-Caucasian differences in nicotine and cotinine metabolism (abstract), Clinical Pharmacology and Therapeutics, 57, 159.

Carmelli, D., Swan, G. E., Robinette, D. \& Fabsitz, R. (1992) Genetic influence on smoking: a study of male twins, New England fournal of Medicine, 327, 829-833.

Dale, H. H. (1914) The action of certain esters and ethers of choline, and their relation to muscarine, foumal of Pharmacology and Experimental Therapeutic$s, 6,147-190$.

EMERICH, D. F., ZaNol, M. D., Norman, A. B., MCConville, B. J. \& SANBerg, P. R. (1991) Nicotine potentiates haloperidol-induced catalepsy and locomotor hypoactivity. Pharmacology, Biochemistry $\mathcal{F}$ Behavior, 38, 875-880.

Freedman, R, AdleR, L. E., Bickford, P. et al. (1994) Schizophrenia and nicotinic receptors, Harvard Review of Psychiatry, 2, 179-192.

Garvey, D. S., Wasicak, J. T., Decker, M. W. et al. (1994) Novel isoxazoles which interact with brain cholinergic channel receptors have intrinsic cognitive enhancing and anxiolytic activities, fournal of Medicinal Chemistry, 37, 1055-1059.

Heath, A. C., Madden, P. A., Slutske, W. S. \& MarTIN, N. G. (1995) Personality and the inheritance of smoking behavior: a genetic perspective, Behavior Genetics, 25, 103-117.

HENNINGFIELD, J. E. \& BeNOWITZ, N.L. (1995) Cigarettes and addiction, British Medical fournal, $310,1082-1083$.

HenNingField, J. E., KozLowsKi, L. T. \& BenowtTZ, N. L. (1994) A proposal to develop meaningful labeling for cigarettes, fournal of the American Medical Association, 272, 312-314.

Hughes, J. R. (1993) Pharmacotherapy for smoking cessation: unvalidated assumptions, anomalies, and suggestions for future research, fournal of Consulting and Clinical Psychology, 61, 751-760.
Kozlowski, L. T. \& Henningfield, J. E. (1995) Thinking the unthinkable: the prospect of regulation of nicotine in cigarettes by the United States government, Addiction, 90, 165-167.

LANGLEY, J. H. (1905) On the reaction of cells and of nerve-endings to certain poisons, chiefly as regards the reaction of striated muscle to nicotine and curare, Foumal of Physiology, 33, 374-413.

Marks, M. J., Campbell, S. M., Romm, E. \& Collins, A. C. (1991) Genotype influences the development of tolerance to nicotine in the mouse, foumal of Pharmacology and Experimental Therapeutics, 259, 392-402.

MCClEARN, G. E. (1993) Behavioral genetics: the last century and the next, Plomin, R. \& MCClearN, G. E. (Eds) in: Nature, Nuture and Psychology (Washington, DC, American Psychological Association).

Nagata, K., Shinohara, T., Kanno, I., Hatazawa, J. \& Domino, E. F. (1995) Effects of tobacco cigarette smoking on cerebral blood flow in normal adults, in Domino, E. F. (Ed.) Brain Imaging of Nicotine and Tobacco Smoking, pp. 95-108. (Ann Arbor MI, NPP Books).

Newhouse, P. A., Potter, A., Corwin, J. \& Lenox, R. (1994) Age-related effects of the nicotinic antagonist mecamylamine on cognition and behavior, Neuropsychopharmacology, 10, 93-107.

Newhouse, P. A., Potter, A. \& Lenox, R. H. (1993) The effects of nicotinic agents on human cognition: possible therapeutic applications in Alzheimer's and Parkinson's diseases, Medicinal Chemistry Research, 2, 628-642.

Noble, E. P., ST. Jeor, S. T., Rrtchie T. et al. (1994) D2 dopamine receptor gene and cigarette smoking: a reward gene? Medical Hypotheses, 42, 257-260.

Peto, R., Lopez, A. D., Boreham, J., Thun, M. \& Heath, C. (1992) Mortality from tobacco in developed countries: indirect estimates from national vital statistics, Lancet, 339, 1268-1278.

Porchet, H. C., Benowitz, N. L., Sheiner, L. B., \& Copeland, J. R. (1987) Apparent tolerance to the acute effect of nicotine results in part from distribution kinetics, fournal of Clinical Investigation, 80, 1466-1471.

Porchet, H. C., Benowitz, N. L., \& Sheiner, L. B. (1988) Pharmacodynamic model of tolerance: application to nicotine, fournal of Pharmacology and Experimental Therapeutics, 244, 231-236.

SHIFFMAN, S. (1993) Smoking cessation treatment: any progress? Foumal of Consulting and Clinical Psychology, 61, pp. 718-728.

Silver, A. A. \& SANBERG, P. R. (1993) Transdermal nicotine patch and potentiation of haloperidol in Tourette's syndrome, Lancet, 342, 182.

Silverstein, M. D., LAShNER, B. A., \& Hanauer, S. B. (1994) Cigarette smoking and ulcerative colitis: a case-control study, Mayo Clinic Proceedings, 69, 425429.

Silverstein, M. D., Lashner, B. A., Hanauer, S. B., Evans, A. A. \& Kirsner, J. B. (1989) Cigarette smoking in Crohn's disease, American fournal of Gastroenterology, 84, 31-33.

SLADE, J. (1995) Are tobacco products drugs? Evidence from U.S. Tobacco, Tobacco Control, 4, 1-2. 